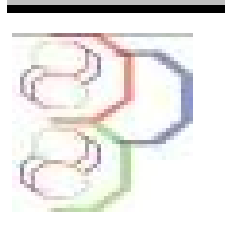

\title{
Impacts des actions anthropiques sur les zones humides de la ville de Douala et solutions de gestion durable : cas de la rivière Kondi
}

\author{
Nwamo Roland Didier*1,3, Ba'ana Etoundi Marie Louise², Tchoumbougnang François ${ }^{1,3}$, Dibong Didier \\ Siegfried ${ }^{1,3}$ \\ 1. Institut des Sciences Halieutiques, Université de Douala, B.P. 2701 Douala, Cameroun \\ 2. Département de Géographie, Faculté des Lettres et Sciences Humaines, Université de Douala, B.P. 2701 Douala, \\ Cameroun \\ 3. Faculté des Sciences, Université de Douala, B.P. 2701 Douala, Cameroun \\ Corresponding author: *nwamodidier@gmail.com
}

Original submitted in on 19th January 2016. Published online at www.m.elewa.org on $31^{\text {st }}$ March 2016 http://dx.doi.org/10.4314/jab.v99i1.8

\begin{abstract}
RESUME
Objectif : Les observations faites au Cameroun notamment dans la ville de Douala montrent que, l'état des écosystèmes aquatiques où sont déversées les eaux résiduaires provenant des différents dépotoirs des industries et des agglomérations et même du lessivage des terres environnantes, est assez démonstratif de l'impact des activités anthropiques. L'objectif du présent travail est d'inventorier les différentes formes d'anthropisation, ainsi que leurs effets sur le milieu, afin de proposer des solutions de gestion durable.

Méthodologie et résultats : La méthodologie adoptée est celle basée sur les enquêtes. Les types d'enquêtes adoptés sont les enquêtes face à face et par observation directe. Les résultats des enquêtes ont montré que plus de la moitié des riverains de la rivière Kondi se sont installées dans cette zone à cause des difficultés financières ont été averties des menaces éventuelles au moment de leur installation sur ce site. L'inondation apparaît comme la première menace subie par les populations. L'élevage et la construction des maisons en bordure du cours d'eau sont les activités anthropiques à l'origine des catastrophes le long du cours d'eau. De manière générale, les populations affirment déverser leurs déchets dans les bacs à ordures et la majorité des domiciles ne possèdent pas des latrines aménagées. Par ailleurs, plus de la moitié de la population connait les noms des espèces végétales et les utilise à des fins alimentaire et sanitaire. Enfin, la proposition du drainage du cours d'eau est la plus avancée par les populations riveraines.

Conclusion et application des résultats : L'analyse des données obtenues a permis de recenser différentes formes d'activités anthropiques menées par les populations et, d'en évaluer leurs conséquences. Les propositions de gestion durable découlant de ces effets donnent une indication sur la perception qu'ont les populations sur la qualité des milieux aquatiques de la ville de Douala, et une orientation de l'assainissement des cours d'eau.
\end{abstract}

Mots clés : Rivière Kondi, écosystème aquatique, activités anthropiques 


\title{
Nwamo et al. J. Appl. Biosci. 2016 Impacts des actions anthropiques sur les zones humides de la ville de
} Douala et solutions de gestion durable : cas de la rivière Kondi.

Impacts of human actions on the wetlands of the city of Douala and sustainable management solutions: case of Kondi River

\begin{abstract}
Objectives: Observations in Cameroon including Douala show that the status of aquatic ecosystems where wastewater discharged from various dumps industries and cities and even leaching of surrounding land, is quite demonstrative of impact of human activities. The objective of this work is to inventory the various forms of human impact and their effects on the environment, to propose sustainable management solutions.

Methodology and results: The methodology is based on the investigations. The kinds of surveys are adopted inquiries face to face and by live observation. Survey results showed that more than half of the residents of the river into this area Kondi moved because of financial and difficulties were warned of feasible threats at their installation on that site; appears the flood as the first threat faced by populations. Farming and building houses on the edge of the river are the anthropogenic activities causing disaster along the river. In general, people say their dump waste in the garbage bins and the majority of homes-have only improved latrines. Furthermore, over half of the population know the names of plants and used in food and health Purposes. Finally, river drainage of the proposal is the most advanced by local residents.

Conclusion and application of results: The analysis of data has identified different forms of human activities and populations assess their consequences. The sustainable management proposals resulting from these effects give an indication of the perception of people about the quality of aquatic environments of the city of Douala, and guidance for remediation of waterways.
\end{abstract}

Keywords: Kondi River, aquatic ecosystem, human activities

\section{INTRODUCTION}

La population mondiale en général et africaine en particulier a enregistré une explosion démographique considérable au cours de ces dernières années (Fresco, 2003). Cette croissance démographique s'accompagne d'un besoin équivalent en production alimentaire, en surface des terres exploitables et en eau. Les eaux de surface occupent la plus grande partie du globe terrestre et environ $98 \%$ de ces eaux sont des eaux marines. Les $2 \%$ restants constituent les eaux continentales représentées par les fleuves, les rivières, les lacs, les étangs ... Ces eaux continentales sont d'une très grande importance pour l'Homme à cause de leurs utilisations multiples. Par ailleurs, les milieux aquatiques continentaux procurent une variété de biens et de services à l'Homme, leur confèrent une valeur économique irremplaçable (Gleick, 1993; Costanza et al. 1997). La primauté de ces services revient à l'eau potable qui est vraisemblablement le bien le plus précieux, car elle est une ressource rare et vitale (Gleick, 1993). L'eau est également un élément indispensable utilisé pour l'irrigation agricole, la production d'énergie et l'industrie. Au cours du Sommet Mondial sur le Développement Durable (SMDD) tenu à Johannesburg en 2002, un constat global s'est dégagé à savoir l'amenuisement progressif des ressources en eau mobilisable lié au développement industriel, urbain, et agro - sylvo pastoral. (Voctabeua, 2013). A ceux-ci, s'ajoutent une forte croissance démographique accompagnée d'une augmentation sans cesse des besoins en eau de bonne qualité. Le Cameroun, pays d'Afrique subsaharienne n'en est point exclu, malgré le fait qu'il possède une importante quantité de ressources en eau. Plusieurs plans d'eau du pays, y compris des zones humides dont la diversité biologique est reconnue d'importance mondiale sont ainsi asphyxiés. Selon l'article I de la Convention de Ramsar de 1971, les zones humides sont des étendues de marais, de fagnes, de tourbières, d'eaux naturelles ou artificielles, permanentes ou temporaires, où l'eau est stagnante ou courante, douce, saumâtre ou salée, y compris des étendues d'eau marine dont la profondeur à marée basse n'excède pas six mètres. (Convention de Ramsar, 2013). Ainsi, dans une zone humide, le principal facteur qui influence le biotope et la biocénose est l'eau. Mais, depuis quelques années, dans un contexte d'urbanisation croissante et de politiques agricoles productivistes, les espaces naturels se sont réduits au profit du développement des activités économiques, résidentielles, touristiques (Marion, 


\section{Nwamo et al. J. Appl. Biosci. 2016 Impacts des actions anthropiques sur les zones humides de la ville de}

Douala et solutions de gestion durable : cas de la rivière Kondi.

2009). Parmi ces espaces naturels, les zones humides, garantes de la biodiversité du territoire et de l'autoépuration des milieux aquatiques, ont été à plusieurs reprises, remises en cause par des constructions, des opérations de drainage, d'assèchement ou de curage, etc. (Marion, 2009). Ainsi, les eaux continentales attirent et concentrent de nombreuses populations pour leurs activités qui en retour devraient veiller à leur gestion et à leur pérennité. Les activités humaines représentent l'une des causes majeures du stress des écosystèmes aquatiques notamment des zones humides (Dokulil et al., 2000; Tazi et al., 2001). Face à l'explosion démographique actuelle, les ressources en eau douce deviennent épuisables et se dégradent fortement. Cette dégradation découle essentiellement des pollutions ponctuelles et diffuses, de la modification des caractéristiques physico-chimiques et de l'exploitation des surfaces de terres cultivables (Malmqvist et Rundle, 2002). Les causes de dégradation des zones humides sont multiples notamment la croissance démographique, les modèles de développement économique, la gouvernance, la faible place des zones humides dans l'agenda politique et le niveau insuffisant d'application des lois environnementales (OZHM, 2012). Ces différentes actions impactent négativement, non seulement la qualité écologique de l'hydro-système, mais aussi les communautés biologiques en général (Malmqvist et Rundle, 2002 ; Bollache et al., 2004). Les ressources de nombreux

\section{MATERIEL ET METHODES}

Populations cibles : Une enquête a été menée auprès des populations riveraines de la rivière "Kondi » réparties dans six quartiers (Beedi, Cité des Palmiers, Malangue, Ndogbong, Point Kilométrique 8 et Vallée Saint Thomas). Ces localités sont composées d'une diversité ethnique, religieuse et sociale. La majorité de ces populations est à vocation commerçante et agricole. L'enquête a concerné 211 ménages pour un total de 703 soit un pourcentage théorique de $30,01 \%$ des ménages ayant contribué à l'enquête.

Choix du site d'étude: Le choix du site d'étude se justifie par l'absence de travaux scientifiques portant sur les activités anthropiques menées dans la zone humide de Kondi qui, nécessite un plan d'aménagement urgent pour une gestion durable. plans d'eau sont donc irréversiblement mal exploitées et endommagées, surtout celles proches des grandes agglomérations (Zohary et al., 1996). Le Cameroun regorge plusieurs zones humides d'un grand intérêt (pour l'hydroélectricité, l'irrigation, l'agriculture, l'élevage, la pêche, l'écotourisme, l'approvisionnement et l'alimentation en eau). Mais, des observations faites notamment dans la ville de Douala montrent que ces écosystèmes aquatiques sont de plus en plus dégradés par les activités anthropiques. Un tel constat constitue vraisemblablement les premiers symptômes d'une « mauvaise " santé de ces écosystèmes démontrant ainsi la nécessité pour les chercheurs de se pencher sur une telle question. Quelques études ont été réalisées sur les répartitions macrophytique et phytoplanctonique des cours d'eau des zones humides de la ville de Douala. C'est le cas des travaux menés par Priso et al. (2000) qui se sont intéressés à l'utilisation des macrophytes comme descripteurs de la qualité des eaux de la rivière «Kondi » dans la ville de Douala. Toutefois, les aspects ayant traits aux relations existant entre la zone humide de Ndogbong-Kondi et les populations riveraines sont inexistants. C'est pour pallier à cette insuffisance et envisager l'aménagement de cette zone humide, que s'inscrit l'objectif général de ce travail. Les objectifs spécifiques sont: (1) inventorier les différentes formes d'anthropisation; (2) déterminer leurs conséquences sur l'écosystème, et (3) proposer des mesures de gestion durable.

Matériel didactique : Le matériel didactique se résume à une fiche d'enquête se présentant sous forme de questions posées à l'enquêté et dont les réponses sont notées ou cochées par ce dernier sur une feuille de questionnaire. Les questions sont ouvertes et/ou fermées. Les grands points de cette fiche concernent l'identification des ethnies constituant les populations riveraines, la situation foncière, l'utilisation des végétaux et de l'eau ainsi que sa qualité et les activités anthropiques menées par les populations riverains, les risques encourus par celles-ci, ainsi que leur avis sur l'occupation du site.

\section{Méthodologie}

Enquête auprès des ménages: L'enquête a été exécutée du 10 au 15 janvier 2014 par un groupe de 60 


\section{Nwamo et al. J. Appl. Biosci. 2016 Impacts des actions anthropiques sur les zones humides de la ville de Douala et solutions de gestion durable : cas de la rivière Kondi.}

étudiants de l'Institut des Sciences Halieutiques de I'Université de Douala, à Yabassi et, concernait les populations riveraines de la rivière Kondi. Le choix des ménages s'est fait au hasard après comptage systématique. Le dialogue s'est déroulé en langues française, anglaise, bafang, bassa et Bouda suivant la langue maîtrisée par l'enquêté. La connaissance de ces

\section{RESULTATS}

Raisons de l'installation aux abords du cours d'eau : Près de la moitié des riverains se sont installées dans le site d'étude à cause des difficultés financières (pauvreté) alors que seulement $2,8 \%$ de ces personnes s'y sont langues par les étudiants impliqués a rendu facile l'accès aux ménages.

Dépouillement de l'enquête: Après la réalisation de toute l'enquête, le travail a été subdivisé en thème et sous thème. Thème par thème ou question par question, le logiciel Sphynx Plus ${ }^{2}$ a permis d'effectuer un dépouillement automatiquement et un tri à plat.

installées à la suite d'un héritage acquis. En outre, $34,6 \%$ des personnes interrogées disent vivre dans cette zone, en raison du domaine foncier peu coûteux (Fig. 1).

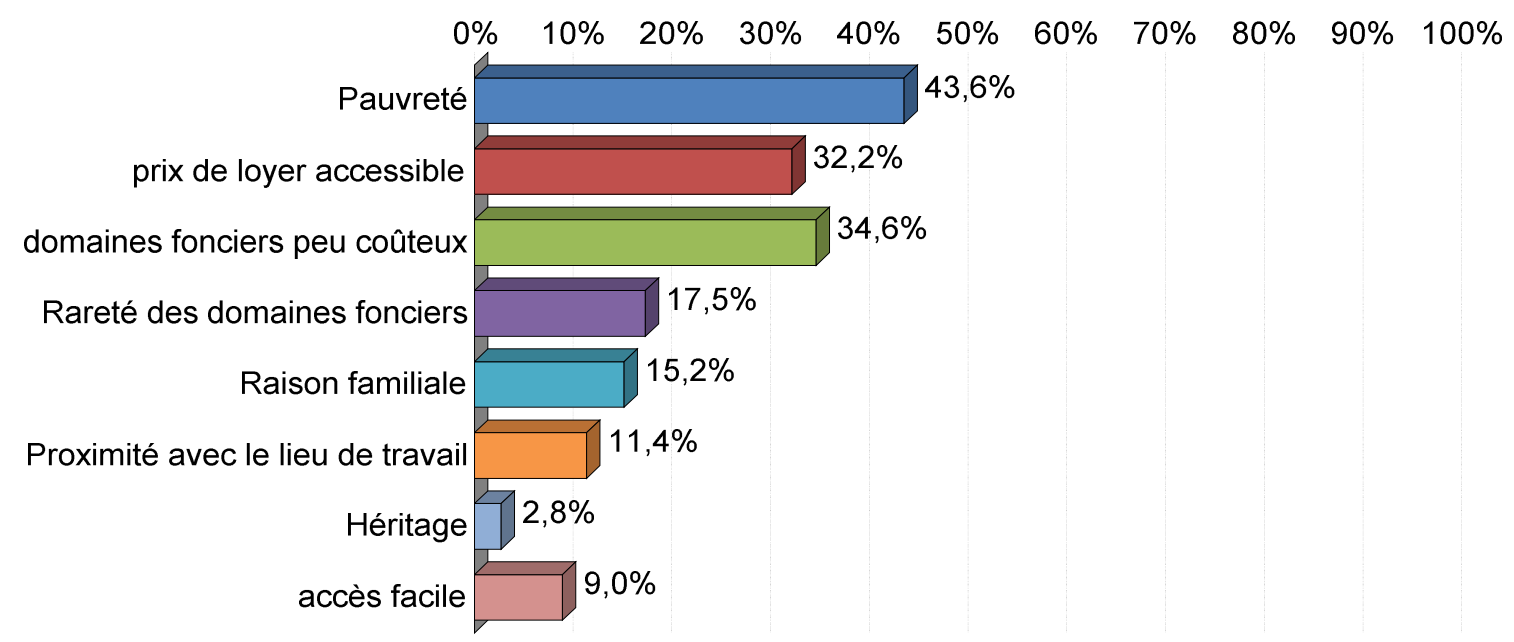

Figure 1 : Raison de l'installation aux abords du cours d'eau

Connaissance des menaces: Parmi les riverains, $63,5 \%$ a été averti des menaces éventuelles au moment de leur installation sur le site. Entre les personnes connaissant les menaces aux quelles elles se sont exposées en s'installant sur ce site, $31,7 \%$ pensent que la pauvreté les a conduit à s'y installer, alors que moins de $5 \%$ de ces personnes disent s'y être installées à cause de l'accès à l'héritage. Parmi les riverains ne connaissant pas les menaces auxquelles ils se sont exposés, $19,7 \%$ ont manqué de moyens financiers, alors que moins de $5 \%$ s'y sont retrouvés à la suite d'un héritage (Fig. 2). Par ailleurs, l'accès facile au site (P.K. 8 et Beedi), le domaine foncier peu coûteux (NdogbongKondi et Vallée Saint Thomas) et le faible coût du loyer (Cité des Palmiers) restent les raisons majeures d'installation de ces populations riveraines. 


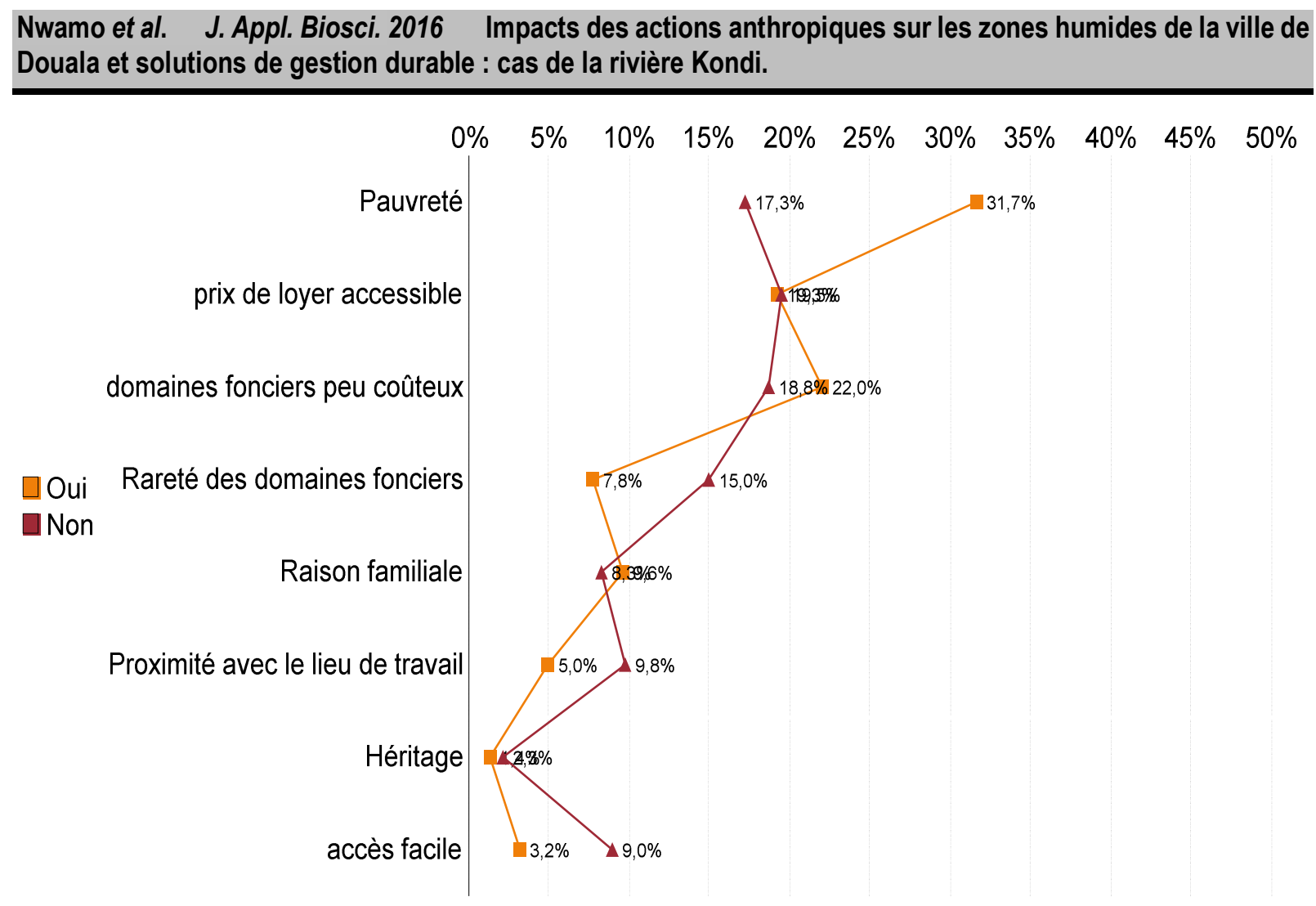

Figure 2 : Connaissance des menaces par les populations

Nature des menaces subies: L'inondation apparaît comme la première menace $(97,8 \%)$ subie par les populations suivis du paludisme $(31,3 \%)$ et aux maladies

hydriques (27,6\%). Par contre, le phénomène d'insécurité reste une menace négligeable pour ces populations (3\%) (Fig. 3).

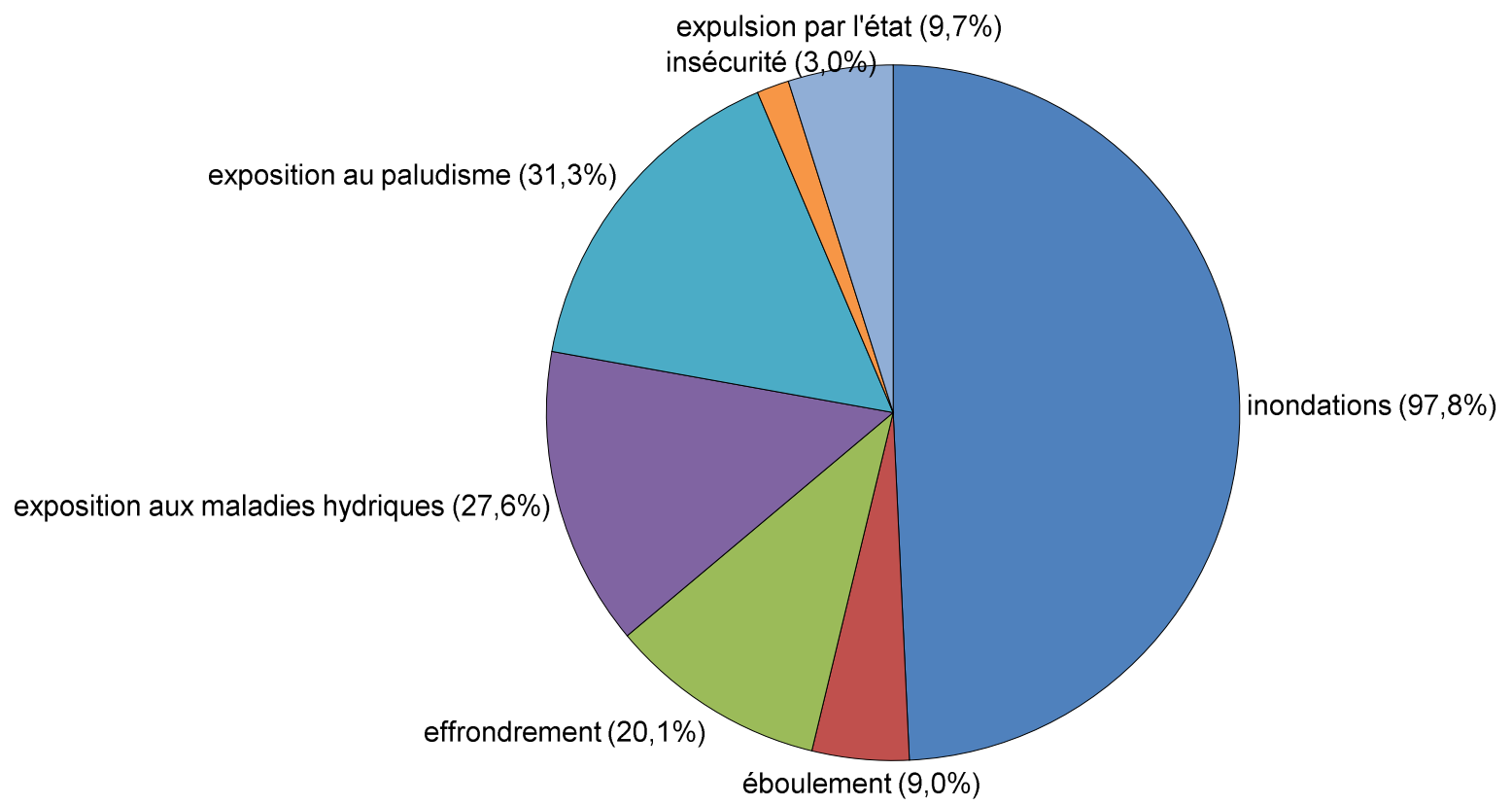

Figure 3 : Nature des menaces subies par les populations riveraines 


\section{Nwamo et al. J. Appl. Biosci. 2016 Impacts des actions anthropiques sur les zones humides de la ville de Douala et solutions de gestion durable : cas de la rivière Kondi.}

Raisons des catastrophes liées aux activités anthropiques : La construction des maisons en bordure du cours d'eau figure parmi les activités anthropiques les plus menées par les personnes ayant déjà été victimes d'une catastrophe $(75,6 \%)$, tandis que la production de la matière plastique se révèle être l'activité qui induit le moins les catastrophes (53,8) (Fig. 4). Pour faire face aux menaces liées aux différentes catastrophes subies par les populations riveraines, ces dernières procèdent au remblai de leur domaine foncier (54,5\%). Par ailleurs, la sensibilisation, le changement de site, ne figurent guère parmi les mesures prises.

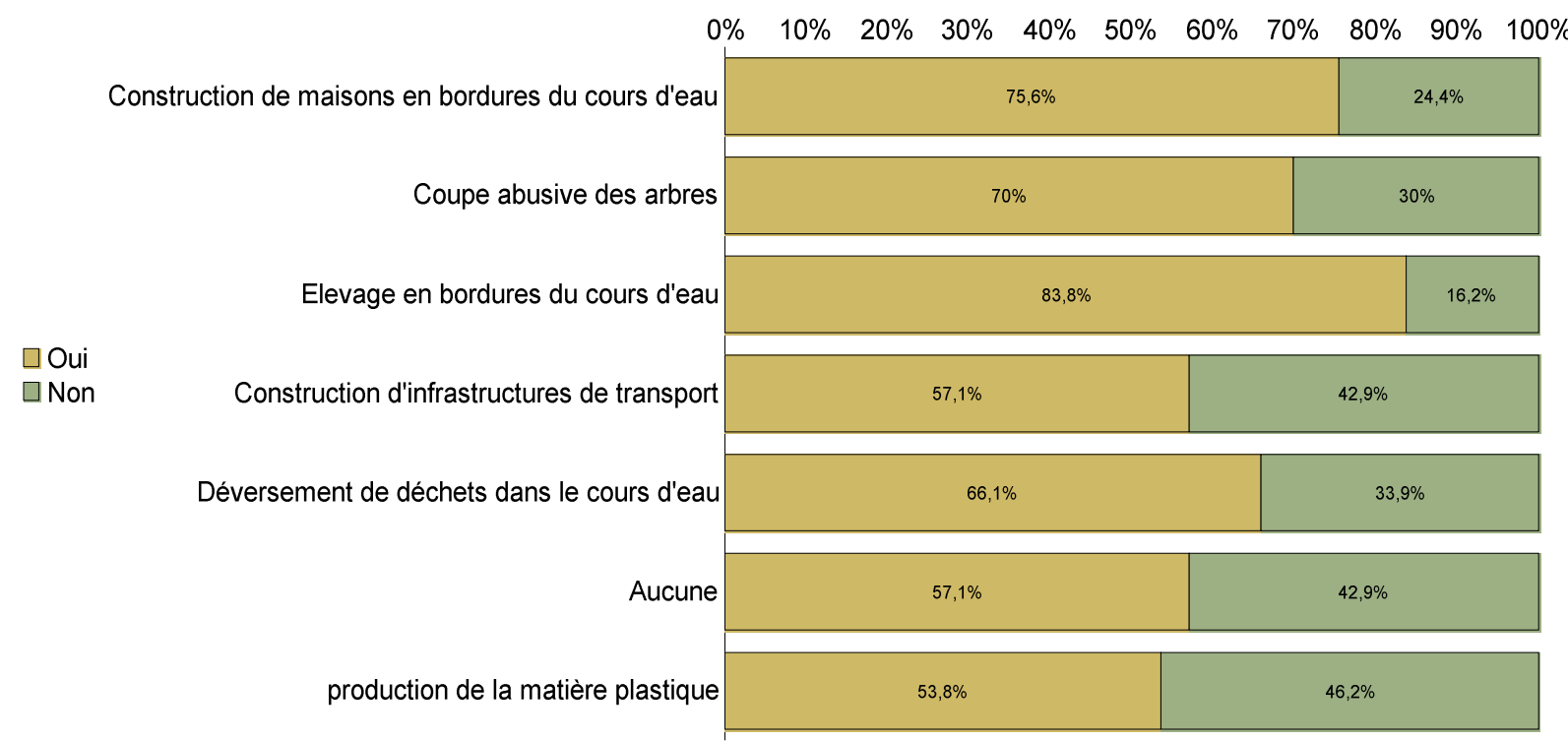

Figure 4 : Raisons des catastrophes en fonction des activités anthropiques

Mesures prises par les populations riveraines pour éviter les catastrophes: Deux grands groupes de quartiers s'opposent par rapport aux mesures prises: celui de Ndogbong-Kondi associé au déménagement temporaire et au drainage du lit du cours d'eau et celui de Malangue/Vallée Saint Thomas où aucune mesure n'est prise (Fig. 5). 


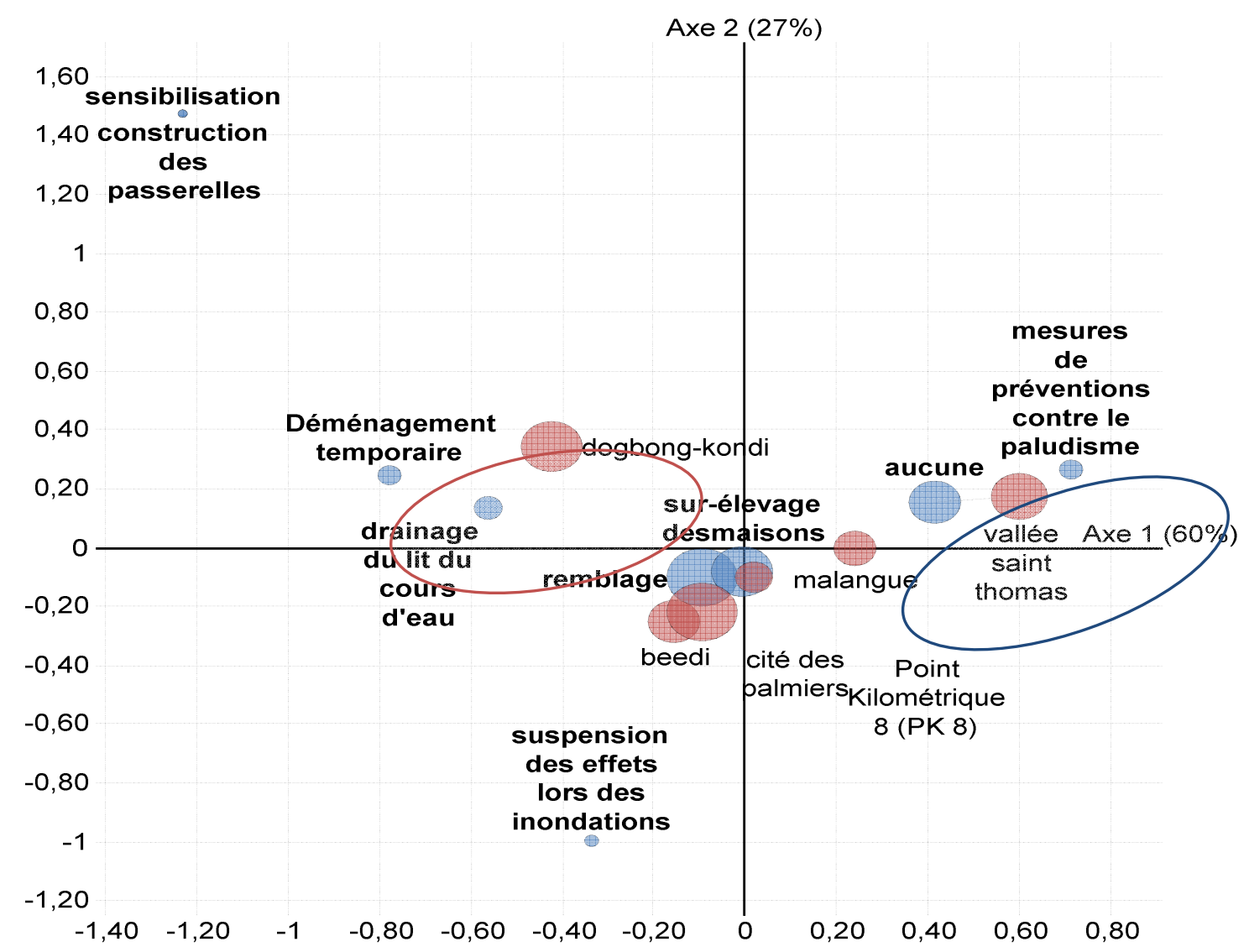

Figure 5 : Représentation de l'AFC des mesures prises par les populations riveraines

Impacts des activités liées à l'homme : L'ampleur des activités liées à l'homme oppose deux groupes de quartiers: le groupe à l'ampleur majeure [30-40\%] (Malangue, Ndogbong-Kondi, Beedi et Vallée SaintThomas) et le groupe à ampleur mineur [10-13,7\%] (Cité des palmiers et P.K. 8). Les activités anthropiques les plus menées sont l'agriculture $(82,5 \%)$ et l'élevage $(17,5 \%)$ et servent à la subsistance des ménages. Les activités de mécanique $(1,8 \%)$ et d'infirmerie $(1,8 \%)$ restent mineures. La majorité des personnes menant une activité la font sur la rive du cours d'eau (56,1\%), alors que $38,6 \%$ s'en éloigne. $3,5 \%$ seulement de ces personnes mènent des activités sur le lit du cours d'eau. $8,5 \%$ des riverains utilisent des produits visant à améliorer leur production agricole ou à détruire des espèces végétales invasives, alors que $18,5 \%$ d'elles prétendent le contraire. Le changement de couleur de l'eau de la rivière serait dû à l'augmentation de l'activité industrielle selon $82 \%$ des personnes interrogées. L'eau de la rivière Kondi n'est pas consommée de façon directe par les populations riveraines. Néanmoins, $41,7 \%$ de ces personnes l'utilise pour leur activité agricole. La majorité des personnes affirment déverser leurs déchets dans les bacs à ordures $(62,6 \%)$, alors qu'environ 12 et $30 \%$ de celles-ci affirment respectivement déverser leurs déchets aux abords du cours d'eau et à l'intérieur de celui-ci. La plupart des domiciles possèdent des latrines non aménagées situées sur la rive et $89,10 \%$ de la population connait les noms des espèces végétales et les utilise à des fins alimentaire et sanitaire.

Utilisation des espèces végétales spécifiques : Parmi les espèces connues par les populations riveraines, aucune d'elles n'est utilisée pour l'activité de pêche. Zea mays est l'espèce la plus utilisée pour la consommation $(19,7 \%)$ et l'agriculture (25\%), tandis que Cymbopogon citratus est l'espèce la moins connue. Pennisetum sp. est plus utilisé dans le secteur agricole alors que Vernonia amygdalina l'est plus dans l'alimentation. En santé, Eremomastax speciosa est l'espèce la plus utilisée. (Fig. 6) 


\section{Nwamo et al. J. Appl. Biosci. 2016 Impacts des actions anthropiques sur les zones humides de la ville de Douala et solutions de gestion durable : cas de la rivière Kondi.}

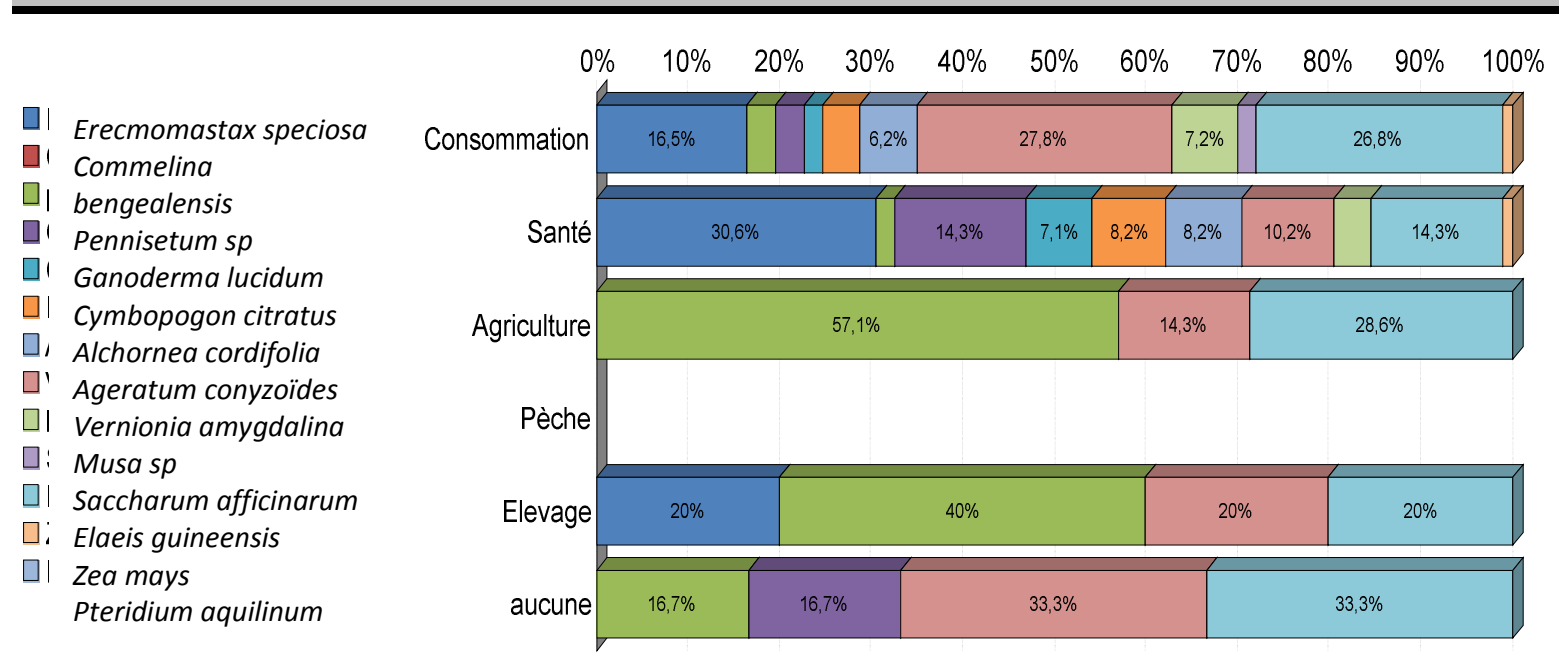

Figure 6 : Utilisations des espèces végétales

Propositions de gestion durable : Parmi les solutions proposées en rapport avec la gestion durable du site étudié, le drainage du cours d'eau $(93,4 \%)$ et l'évitement des constructions sur le lit du cours d'eau $(37,9 \%)$ sont les plus évoquées (Fig. 7).

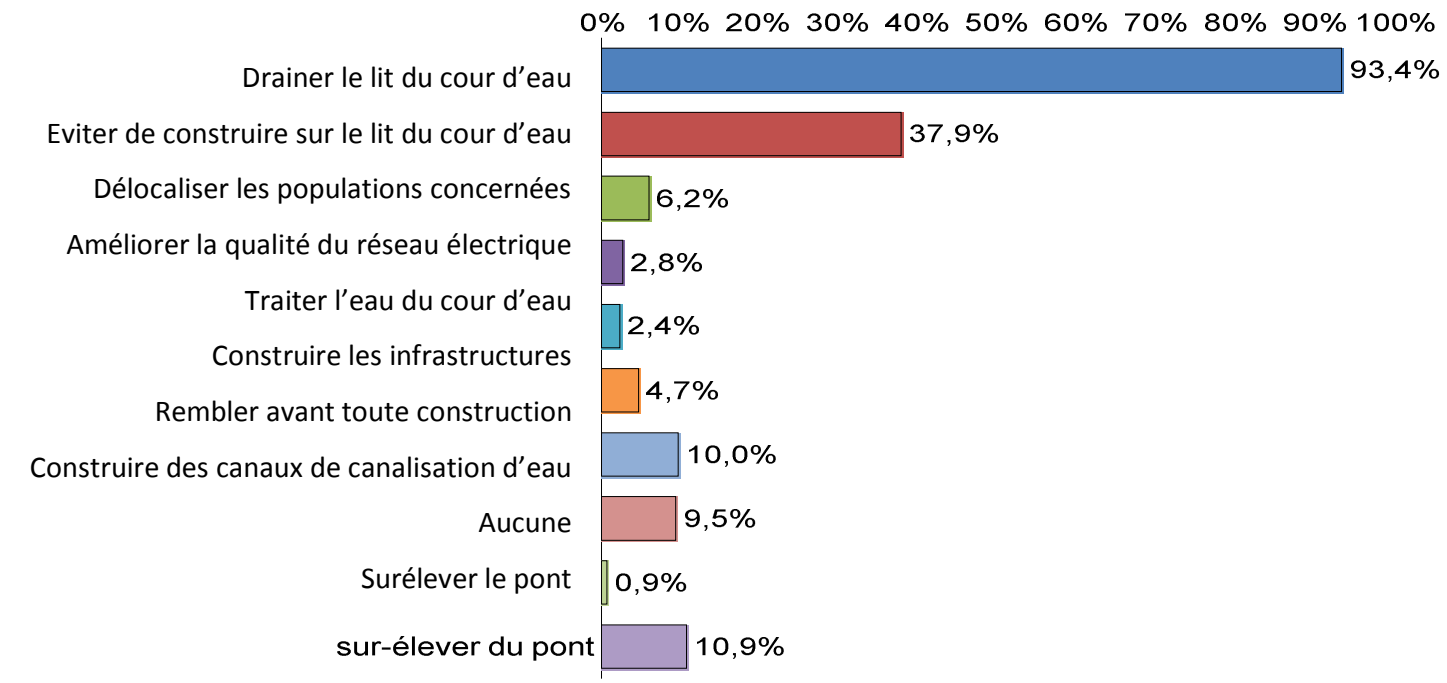

Figure 7 : Propositions de gestion durable

\section{DISCUSSION}

La majorité des personnes $(43,6 \%)$ se sont installées dans le site étudiée à cause de la pauvreté. En outre, $34,6 \%$ des personnes disent vivre dans cette zone en raison du domaine foncier peu coûteux. $63,5 \%$ pensent que la faiblesse des prix de vente des parcelles est évocatrice. Ces résultats sont différents de ceux obtenus par Oumou et al., (2009) qui ont démontré que $73 \%$ des populations habitant la zone humide du Diawling en Mauritanie sont pauvres, avec un profil de pauvreté dressé. Mais dans le cas présent, le profil de pauvreté n'a pu être dressé en raison de la non incorporation des questions relatives aux revenus et dépenses des ménages enquêtés. Entre les choix de réponses soumis aux riverains de la rivière Kondi, l'inondation apparaît comme la première menace $(97,8 \%)$ subie par les populations, suivies du paludisme et des maladies hydriques. Ces résultats se rapprochent des données de I'IRSERM (2015), qui affirment que le paludisme sévit depuis des millénaires dans les zones marécageuses de l'ensemble du globe. Ces résultats sont également confortés par les conclusions tirées par Vasquez et Favila, (1998); Dokulil et al. (2000) et Tazi et al. (2001). 


\section{Nwamo et al. J. Appl. Biosci. 2016 Impacts des actions anthropiques sur les zones humides de la ville de Douala et solutions de gestion durable : cas de la rivière Kondi.}

Selon ces auteurs, les activités humaines représentent l'une des causes majeures du stress des écosystèmes aquatiques. La construction des maisons en bordure du cours d'eau figure parmi les activités anthropiques les plus menées par les personnes ayant déjà été victime d'une catastrophe $(75,6 \%)$. L'ensemble des riverains ayant observés un changement de couleur de l'eau révèlent à $82 \%$ que cette situation serait due à l'augmentation de l'activité industrielle qu'à l'augmentation de la population $(11,2 \%)$. Ces résultats sont confortés par les conclusions tirées par Dokulil et al. (2000) et Tazi et al. (2001). Selon ces auteurs, les activités humaines représentent l'une des causes majeures du stress des écosystèmes aquatiques. Au contraire, l'OZHM (2012), pense que si l'agriculture est le secteur qui impacte le plus les zones humides et l'eau, l'urbanisation, les infrastructures publiques et le tourisme exercent des pressions croissantes. Pour faire face aux menaces liées aux différentes catastrophes, les populations riveraines du Kondi procèdent le plus souvent au remblai de leur domaine foncier. Par ailleurs, la sensibilisation, le changement de site, le déménagement temporaire et la construction des passerelles ne figurent guère parmi les mesures prises par ces populations pour prévenir les catastrophes. Parmi les activités menées, l'agriculture $(82,5 \%)$ et l'élevage $(17,5 \%)$ occupent les premiers rangs. Ces données sont différentes de celles trouvées par Oumou et al.. (2009), qui ont démontré que $26,7 \%$ des populations d'une zone humide en Mauritanie exercent une activité économique. Néanmoins, l'élevage qui se situe dans le lot des activités secondaires corrobore les résultats obtenus. La majorité des personnes effectuant une activité la fait sur la rive du cours d'eau Kondi (56, $5 \%$ ), alors que $3,1 \%$ des riverains affirment mener leurs activités dans le cours d'eau. 8,5\% des personnes disent utiliser des produits visant à améliorer leur production agricole ou à détruire des espèces végétales invasives. Ces résultats sont confortés par Malmqvist et Rundle (2002) pour qui la dégradation des ressources en eau douce découle essentiellement des pollutions ponctuelles et diffuses, de la modification

\section{CONCLUSION}

Les enquêtes ont montré que plus de la moitié des personnes se sont installées dans cette zone à cause des difficultés financières. Celles-ci ont été averties des menaces éventuelles au moment de leur installation. L'inondation apparaît comme la première menace subie par les populations. La majorité des populations se sont appuyées sur l'accès facile au site et le faible coût du domaine foncier pour s'y installer. Parmi les personnes des caractéristiques physico-chimiques et de l'exploitation des surfaces de terres cultivables dues à l'intensification des activités agricoles. Les données de l'enquête relative à l'utilité de l'eau de la rivière Kondi démontrent qu'aucune des personnes ne consomme cette eau de façon directe. Néanmoins, $41,7 \%$ de celle-ci l'utilise pour l'irrigation agricole. Ces résultats sont différents de ceux publiés par Gleick (1993), pour qui la primauté de ces services revient à l'eau potable qui est vraisemblablement le bien le plus précieux, car elle est une ressource rare et vitale. La majorité des personnes affirment déverser leurs déchets dans les bacs à ordures $(62,6 \%)$ alors que $30,0 \%$ et $14,1 \%$ de celles-ci affirment respectivement déverser leurs déchets aux abords du cours d'eau et à l'intérieur de celui-ci. Ce déversement de déchets stresse ainsi le cours d'eau qui les accueille. De plus, les domiciles des riverains possèdent en majorité des latrines situées sur de la rive. On constate donc une différence avec les résultats de Oumou et al en 2009 sur l'assainissement (position des latrines) dans la zone humide du Diawling en Mauritanie, par le fait qu'à l'issue de leur étude, $82 \%$ de ménages ne disposaient pas d'installations sanitaires adéquates. Parmi les personnes, $89,10 \%$ d'elles connaissent les noms des espèces végétales herbacées et les utilisent à des fins alimentaire et médicinale. Toutes les espèces végétales citées par les populations riveraines (Cymbopogon citratus, Eremomastax speciosa, Pennisetum sp., Vernomia amygdalina, Zea mays), sont des herbacées ayant une importance médicinale comme l'attestent les travaux de Mpondo Mpondo et Dibong (2011) et de Ndjouondo et al. (2015). Le drainage du cours d'eau, plus avancé par les riverains $(93,4 \%)$ semble être la solution adéquate, selon Marion (2009). En effet, elle révèle que, parmi les espaces naturels exploités, les zones humides, espaces de transition entre la terre et l'eau, garantes de la biodiversité du territoire et de l'autoépuration des milieux aquatiques, ont été à plusieurs reprises, remises en cause par des constructions, des opérations de drainage, d'assèchement ou de curage.

ayant trouvées le terrain marécageux, plusieurs d'entre elles ont déjà été victimes d'une catastrophe. Le déversement des déchets dans le cours d'eau figure parmi les activités anthropiques les plus menées par les personnes ayant déjà été victimes d'une catastrophe le long du Kondi. Pour faire face aux menaces liées aux différentes catastrophes subies par les populations, ces dernières procèdent au remblai de leur domaine foncier. 


\section{Nwamo et al. J. Appl. Biosci. 2016 Impacts des actions anthropiques sur les zones humides de la ville de Douala et solutions de gestion durable : cas de la rivière Kondi.}

Par ailleurs, les activités les plus menées sont l'élevage et l'agriculture et la majorité des personnes effectuant une activité la font sur la rive du cours d'eau. De manière générale, les déchets sont déverser dans les bacs à ordures et la majorité des domiciles ne possèdent pas

\section{RÉFÉRENCES BIBLIOGRAPHIQUES}

Bollache L, Devin S, Wattier R, Chovet M, Beisel JN, Moreteau JC et Rigaud T, 2004. Rapid range extension of the Ponto-Caspian amphipod Dikerogammarus villosus in France: potential consequences. Arch. Hydrobiologie 160 : 57-66.

Constanza R, d'Arge R, Rudolf de Groot, Stephen Fk Monica G, Bruce H, Karin L, Shahid NRV O'Neill, Jose P, Robert GR, Paul SK et Marjan van den B, 1997. The value of the world's ecosystem services and natural capital in Nature's Services vol 387.

Convention de Ramsar, 2013. Le Manuel de la Convention de Ramsar: Guide de la Convention sur les zones humides (Ramsar, Iran, 1971), 6e édition. Secrétariat de la Convention de Ramsar, Gland, Suisse.

Dokulil M Chen W et Cai Q, 2000. Anthropogenic impacts to large lakes in China: the Tai Hu example. Aquatic Ecosystem Health and Management 3: 81-94.

Fresco OL, 2003. Les engrais et l'avenir. In : la sécurité mondiale et le rôle de la fertilité durable. Maisonneuve Larousse (eds). Conférence IFAIFAO. Rome. Italie. 26-28 mars 2003 pp. 5167.

Gleick PH, 1993. "Water resources: A long-range global evaluation." Ecology Law Quarterly 20 (1) : pp. 141-149.

IRSERM, 2015. Dossier d'information sur le paludisme, unité de recherche 1135 Inserm/UPMC, Centre d'immunologie et des maladies infectieuses, Paris) - janvier 2015

Marion, 2009. Les zones humides : vers une gestion plus concertée ? p.29

Malmqvist $B$ et Rundle $S, 2002$. Threats to the running water ecosystems of the world. Environmental Conservation p. 29. des latrines aménagées. Plus de la moitié de la population connaissent les noms des espèces végétales et les utilise à des fins alimentaire et sanitaire. Enfin, la proposition du drainage du cours d'eau est celle la plus avancée et la plus appropriées.

Mpondo Mpondo E, Dibong SD, 2011. Medicinal plant knowledge of ethnic groups in Douala town, Cameroon. Am. J. Food. Nutr. 1(4): 178 - 184.

Ndjouondo GP, Ngene J-P, Ngoule CC, Kidik P M-C, Ndjib RC, Dibong SD, Mpondo ME, 2015. Inventaire et caractérisation des plantes médicinales des sous bassins versants Kambo et Longmayagui (Douala, Cameroun). Journal of Animal and Plant Sciences, 25(3) 3898-39.

OZHM., 2012. Rapport sur l'état des zones humides dans le bassin méditerranéen.

Ly Oumou K., et Zein, Sidi A.O.M. 2009. Evaluation économique d'une zone humide: le cas de Diawling, Mauritanie, Gland, Suisse : IUCN, p.70

Priso RJ Taffouo V, Kenne M, Amougou A, et De Sloover $J R$, 2000. A propos de l'utilisation des Commelinaceae comme indicateurs de la qualité des milieux aquatiques. Sci. Technol. Dév. 7(1) : 4-11.

Tazi O, Fahde A et El Younoussi S, 2001. Impact de la pollution sur l'unique réseau hydrographique de Casablanca, Maroc. Sécheresse, 12: 129-134.

Vazquez $G$ et Favila ME, 1998. Status of the health conditions of subtropical Atezea lake. Aquatic Ecosystem Health and Management 1: 245 255.

Voctabeua T., EG, 2013. Etudes d'impacts socioéconomiques de la répartition des infrastructures hydriques dans la tranche urbaine de Ngaoundéré II au Cameroun. Licence en géographiephysique. Université de Ngaoundéré-Cameroun. p.1Zohary T, PaisMadeira AM, Robarts R et Hambright KD, 1996. Interannual phytoplankton dynamics of a hypertrophic african lake. Arch. Hydrobiol 136: 105-126. 